\title{
Giant spin-noise gain enables magnetic resonance spectroscopy of impurity crystals
}

\author{
A. N. Kamenskii, ${ }^{1}$ A. Greilich $\odot,{ }^{1}$ I. I. Ryzhov, ${ }^{2,3}$ G. G. Kozlov,${ }^{3}$ M. Bayer,,${ }^{1,4}$ and V. S. Zapasskii ${ }^{3}$ \\ ${ }^{1}$ Experimentelle Physik 2, Technische Universität Dortmund, 44221 Dortmund, Germany \\ ${ }^{2}$ Photonics Department, St. Petersburg State University, Peterhof, 198504 St. Petersburg, Russia \\ ${ }^{3}$ Spin Optics Laboratory, St. Petersburg State University, Peterhof, 198504 St. Petersburg, Russia \\ ${ }^{4}$ Ioffe Institute, Russian Academy of Sciences, 194021 St. Petersburg, Russia
}

(Received 26 February 2020; revised manuscript received 13 March 2020; accepted 28 May 2020; published 11 June 2020)

\begin{abstract}
We apply spin-noise spectroscopy to the classical objects of electron paramagnetic resonance spectroscopydielectrics with paramagnetic impurities — which seemed to be unsuitable for this technique because of their low specific Faraday rotation (FR) associated with strong (allowed) optical transitions. We show, however, that by detecting the spin noise at the wavelengths of weak (forbidden) transitions (with low regular FR), the signal can be strongly enhanced, provided that the optical transition is broadened inhomogeneously. This spin-noise gain effect, which is controlled by the ratio of the inhomogeneous linewidth to the homogeneous one, relates primarily to the parity-forbidden intraconfigurational transitions of the impurity ions with unfilled inner electronic shells. Specifically, for the $f$ - $f$ transitions of rare-earth ions in crystals, this factor can reach $\sim 10^{8}$. In this paper, we describe the specific features of the experimental equipment required for the realization of this kind of measurements, present results of successful experiments of spin-noise spectroscopy on rare-earth ions in crystals, and briefly discuss the prospect of applying this method of magnetic resonance spectroscopy in present-day research.
\end{abstract}

DOI: 10.1103/PhysRevResearch.2.023317

\section{INTRODUCTION}

The spectroscopy of magnetic resonance can be applied to virtually all quantum objects possessing angular momentum and, therefore, is one of the key techniques of contemporary physics research. Detection of the magnetic resonance spectrum usually implies observation of the response of a paramagnet ("spin system") to an AC magnetic field with a frequency close to that of the spin precession. Since the discovery of the magnetic resonance effect by Zavoisky in 1944 [1], two main detection methods were elaborated. One of them uses detection of the changes of the AC field, as done, e.g., in the conventional optical spectroscopies of absorption or reflection. In the second method, the resonance is revealed in the AC-field-induced changes of the object's properties as done, e.g., in optical pump-probe spectroscopy. However, the principle of resonance detection in both cases is the same: the resonance is observed only through the response to the excitation of spin precession by the external AC field.

In 1981 [2], it was demonstrated experimentally that observation of the magnetic resonance does not necessarily require application of the resonant $\mathrm{AC}$ field; the resonance can be observed also by detecting the excess fluctuations of the medium's refractive index at the spin-precession frequency.

Published by the American Physical Society under the terms of the Creative Commons Attribution 4.0 International license. Further distribution of this work must maintain attribution to the author(s) and the published article's title, journal citation, and DOI.
During recent years, this spin-noise spectroscopy (SNS) has considerably developed [3-7] and demonstrated some specific capabilities that were not foreseen initially [8]. Specifically, the SNS sensitivity was found to be sufficient to detect the spin noise $(\mathrm{SN})$ of a single charge carrier in a semiconductor structure $[9,10]$, was applied to monitor the nuclear dynamics of the host lattice in a doped semiconductor [11,12], was used as an instrument of high-resolution tomography [13] and as a spectroscopic tool to identify the origin of broadening of optical transitions and to measure their homogeneous width $[14,15]$, etc. As a result, SNS has nowadays turned into an important method of research in atomic and semiconductor physics [16-19].

It should be noticed, however, that this method has so far not been applied to dielectrics with paramagnetic impuritiessystems which served as the primary material basis for electron resonance (EPR) spectroscopy and are nowadays widely used in state-of-the-art optics and photonics [20-23]. These objects so far did not look promising for the application of SNS because of their low efficiency of conversion of "magnetization-FR." This fact was confirmed in Ref. [24] using data on regular Faraday rotation (FR) of crystals with rare-earth (RE) ions normalized to a single spin (the so-called Faraday-rotation cross section [25]). We show, however, that the efficiency of the magnetization-FR conversion may drastically differ for regular and stochastic signals.

It is useful to remind one here that the impurities in dielectric materials that are most important for science and applications (such as $\mathrm{Cr}^{3+}$ and $\mathrm{Nd}^{3+}$ in laser media, or the $\mathrm{RE}$ ions in up-converters or quantum counters, in quantum memory schemes, etc.) are usually ions of the transition-metal 
(a)
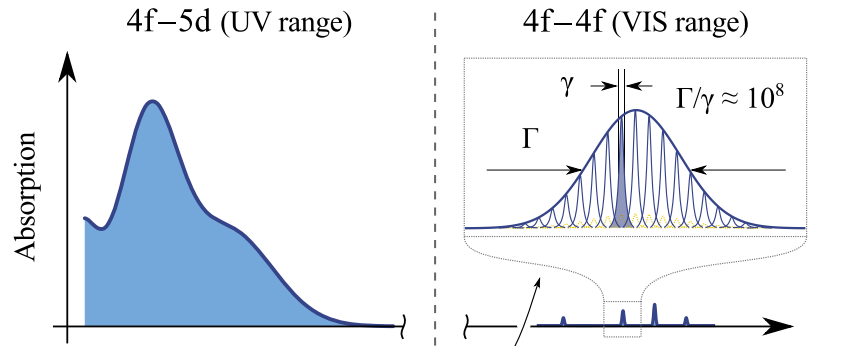

(b)

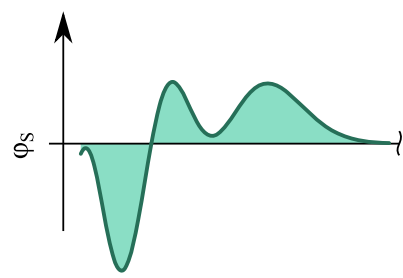

(c)

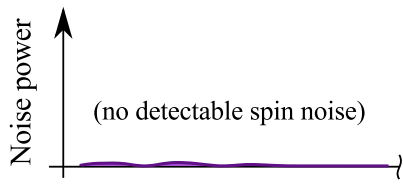

its regular FR (FR in external magnetic field). On the other side, the FR noise appears to be drastically enhanced for the resonantly probed optical transitions with a high ratio of the inhomogeneous linewidth to the homogeneous one [see Fig. 1(c)]. When applied to the forbidden intraconfigurational transitions of RE ions in crystals, this enhancement (the spinnoise gain effect) reaches up to seven to eight orders of magnitude. The proposed experimental approach turns REactivated crystals into favorable objects of SNS.

\section{THEORETICAL CONSIDERATIONS}

It was shown in Ref. [14] that the shape of the optical spectrum (wavelength dependence) of the SN power, detected within the optical transition, is strongly affected by inhomogeneous broadening of the transition. In particular, for a homogeneously broadened line, the optical spectrum of the SN power exhibits a dip in the center of the line which gradually disappears with increasing inhomogeneous linewidth. Here, we will consider the case when the inhomogeneous width of the transition strongly exceeds its homogeneous width and will concentrate on the effect of $S N$ gain revealed under these conditions.

Let us consider the formation of the SN signal under these conditions and derive a simple estimate of the strength of the SN signal. Figures 1(a) and 1(b) show schematically absorption and FR spectra of trivalent paramagnetic RE impurities in crystals in the range of interconfigurational $(f-d)$ and intraconfigurational $(f-f)$ transitions. Let the inhomogeneous and homogeneous linewidths of a single $f$ - $f$ transition be $\Gamma$ and $\gamma$, respectively, as shown in the zoomed-in insets of Figs. 1(a) and 1(b).

It is convenient for our reasoning to choose the wavelength of the probe beam such that the regular FR of the system is essentially different from zero, which is the case somewhere on the slope of the inhomogeneous absorption line. To simplify the calculation, we consider the case of total spin polarization when all spins are oriented along the light propagation direction (say, $S_{i}=+1 / 2$ for all $i$ ) and the magnitude of the FR $\varphi_{s}$ (FR of saturation) is the greatest. Let us express the angle $\varphi_{s}$ through the length of the sample $d$ and impurity concentration $n$ (spin density) using the definition of the FR cross section $\sigma$ introduced in Ref. [25]: $\varphi_{s}=\sigma n d$. The number of impurity centers within the probe beam volume $N$ is given by $N=n d A$, where $A$ is the beam cross section.

Because of the inhomogeneous broadening of the line, the FR (being itself independent of the homogeneous width $\gamma$ ) is contributed only by the fraction of impurity centers with optical frequencies within an interval of the order of the homogeneous linewidth $\gamma$. This number $N^{\prime}$, is approximately equal to $N \gamma / \Gamma$, and thus can drastically differ from the total number of impurity centers $N$.

Now, assuming that the total FR is the sum of contributions of the individual centers, we can write the FR per unit center $\varphi_{i}$ as $\varphi_{i}=\varphi_{s} / N^{\prime} \sim(\Gamma / \gamma) \varphi_{s} / N$. When detecting the $F R$ noise (rather than the standard FR), the magnitude of the contribution of each center will remain $\varphi_{s} / N^{\prime}$ as before, but its sign will be random: $\varphi_{i}= \pm(\Gamma / \gamma) \varphi_{s} / N$.

An important quantity for $\mathrm{SN}$ measurements is the mean square of fluctuations of the $\operatorname{FR}\left\langle\delta \varphi^{2}\right\rangle$, where $\delta \varphi \equiv \sum_{i=1}^{N^{\prime}} \varphi_{i}$. 
The quantity $\left\langle\delta \varphi^{2}\right\rangle$ can be written in the form

$$
\left\langle\delta \varphi^{2}\right\rangle=\left\langle\left[\sum_{i=1}^{N^{\prime}} \varphi_{i}\right]^{2}\right\rangle=[\Gamma / \gamma] \varphi_{s}^{2} / N .
$$

In SNS, we typically detect the spectrum of the noise power or its spectral density, with its characteristic value $W=\left\langle\delta \varphi^{2}\right\rangle T_{2}$, where $T_{2}$ is the spin dephasing time. Using Eq. (1), we obtain

$$
W \equiv\left\langle\delta \varphi^{2}\right\rangle T_{2}=\frac{T_{2} \sigma^{2} \Gamma}{\gamma} \frac{n d}{A} \equiv \eta \frac{n d}{A} .
$$

The parameters entering the last fraction of this equation depend on the measurement conditions and, to a certain extent, can be controlled by the experimentalist. The factor $\eta=T_{2} \sigma^{2} \Gamma / \gamma$ can be considered as a characteristic quantity of the studied paramagnetic center or, better to say, of the particular optical transition addressed. It is this parameter that allows one to compare different spin systems from the viewpoint of applicability of SNS. We see that the factor $\Gamma / \gamma$ (which we call the gain factor) can drastically enhance the noise power, leaving the FR cross section unchanged.

Note, that we have not used a specific spectral shape for the single-impurity contribution to the polarimetric signal. The only parameter we use to estimate the number of centers contributing to the signal is the homogeneous spectral width $\gamma$ of the optical resonance of a single impurity. We emphasize that our estimates do not change when the homogeneous optical width becomes smaller than the Zeeman splitting $\left(\gamma<\omega_{L}\right)$ and the spectral features of the optical susceptibility of single impurity become resolvable in opposite circular polarizations: the number of centers contributing to the polarimetric signal for strictly monochromatic probing will still be controlled by the ratio of the homogeneous and inhomogeneous widths of $\gamma / \Gamma$.

The above reasoning shows, in particular, that objects with a relatively large FR cross section $\sigma$ may be less favorable for the SN technique than those with a smaller $\sigma$ but with a larger gain factor $\Gamma / \gamma$. The FR in the region of the $f-d$ transitions, which is more easily observable on divalent ions, is usually larger than the FR near the $f-f$ transitions. At the same time, the $f$ - $d$ transition bands with width of $\sim 10^{13} \mathrm{~Hz}$ are broadened homogeneously, while the lines of the $f-f$ transitions with widths around $10^{10} \mathrm{~Hz}$ [27], are strongly inhomogeneously broadened, as the homogeneous linewidth often is in the range of $10^{2}-10^{3} \mathrm{~Hz}[26,28]$, thus providing spin-noise gain factors of up to $10^{8}$. It is this effect that allows us to solve the problem of $\mathrm{SN}$ detection in activated crystals.

\section{EXPERIMENTAL RESULTS}

Now, let us turn to the demonstration of the SN gain effect. In our experiments, we used two crystals of cubic symmetry with the RE ions possessing $f-f$ transitions in the spectral range accessible with a Ti:sapphire laser used as the source of the probe light. Sample 1 was a $\mathrm{SrF}_{2}$ crystal coactivated with $\mathrm{Nd}^{3+}(0.5 \mathrm{~mol} \%)$ and $\mathrm{Yb}^{3+}(0.15 \mathrm{~mol} \%)$. Sample 2 was a crystal of $\mathrm{CaF}_{2}: \mathrm{Nd}^{3+}(0.1 \mathrm{~mol} \%)$. Both crystals were grown using the Stockbarger technique in graphite crucibles. In these crystals, the trivalent RE ions substitute divalent cations of the host lattice. For this reason, the impurity centers created

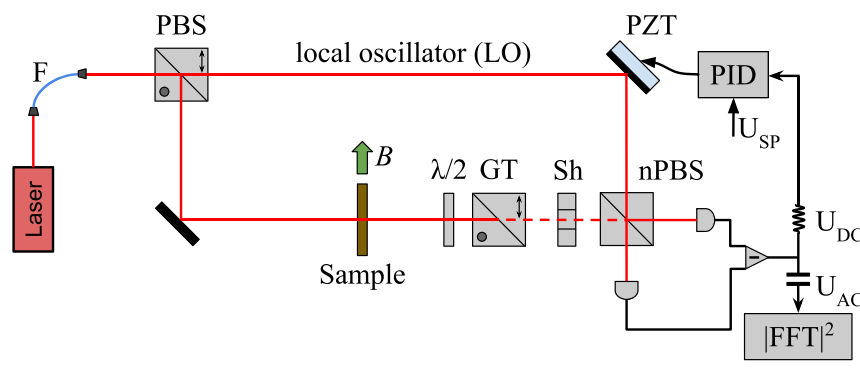

FIG. 2. Experimental schematics. The narrow-band cw laser is guided through a single-mode polarization-maintaining fiber (F). It is then separated into two parts: the local oscillator (LO) and the probe. The probe light is vertically polarized and is sent focused through the sample. The interference of the horizontally polarized scattered light and the LO is measured at the balanced photodiodes. The AC component of the voltage is used to measure the spin-noise power spectrum, and the DC component is used to stabilize the phase of the interference by the PID loop. Here, PBS and nPBS, are polarizing and nonpolarizing beam splitters, respectively. Sh is a mechanical shutter, GT is a Glan-Taylor prism, and $\lambda / 2$ is a half-wave plate. $U_{\mathrm{SP}}$ is the set point voltage for the PID loop of the piezocontrolled mirror (PZT).

thereby are mainly anisotropic due to local compensation of the excess activator charge. As a result, several groups of magnetically nonequivalent centers form and essentially complicate magnetic-resonance spectra by creating several resonances in the $\mathrm{SN}$ spectra at any fixed magnetic field. This situation is typical for the case of heterovalent substitution of impurity ions in cubic crystals [29,30]. Our aim, however, was not to identify and study particular centers in these crystals, but rather to convincingly demonstrate the possibility and efficiency of applying SNS to this class of objects.

To detect the spin noise, we used the homodyne detection scheme (see Fig. 2 [31,32]). It is clear from the consideration above that the SN gain effect can be observed only when the spectral width of the probe laser light is smaller than the homogeneous linewidth $\gamma$. This is why we used a tunable (cw) ring-cavity Ti:sapphire laser with a short-term (during 100 $\mathrm{ms}$ ) spectral width of $<40 \mathrm{kHz}$. When measuring spin noise, the laser was additionally power stabilized and transmitted through a single-mode fiber to symmetrize the spatial mode. Behind the fiber, the laser was coupled into the entrance port of a Mach-Zehnder interferometer, with one beam passing through the sample and the second one bypassing the sample [local oscillator (LO)]. The light scattered by the sample had orthogonal polarization with respect to the incident beam and was then collected and combined with the LO of the interferometer. The resulting interference was measured using balanced photodiodes with $670 \mathrm{MHz}$ bandwidth. The signal was digitized and fast-Fourier transformed by a fieldprogrammable gate array [33]. The normalized difference between the LO with transmitted and blocked scattered probe light was measured for identical external conditions. The sample was placed in a cold-finger He-flow cryostat at a temperature of $T=5.7 \mathrm{~K}$. The magnetic field was generated by an electromagnet in the Voigt configuration, i.e., it was oriented orthogonal to the probe beam direction. The laser was 

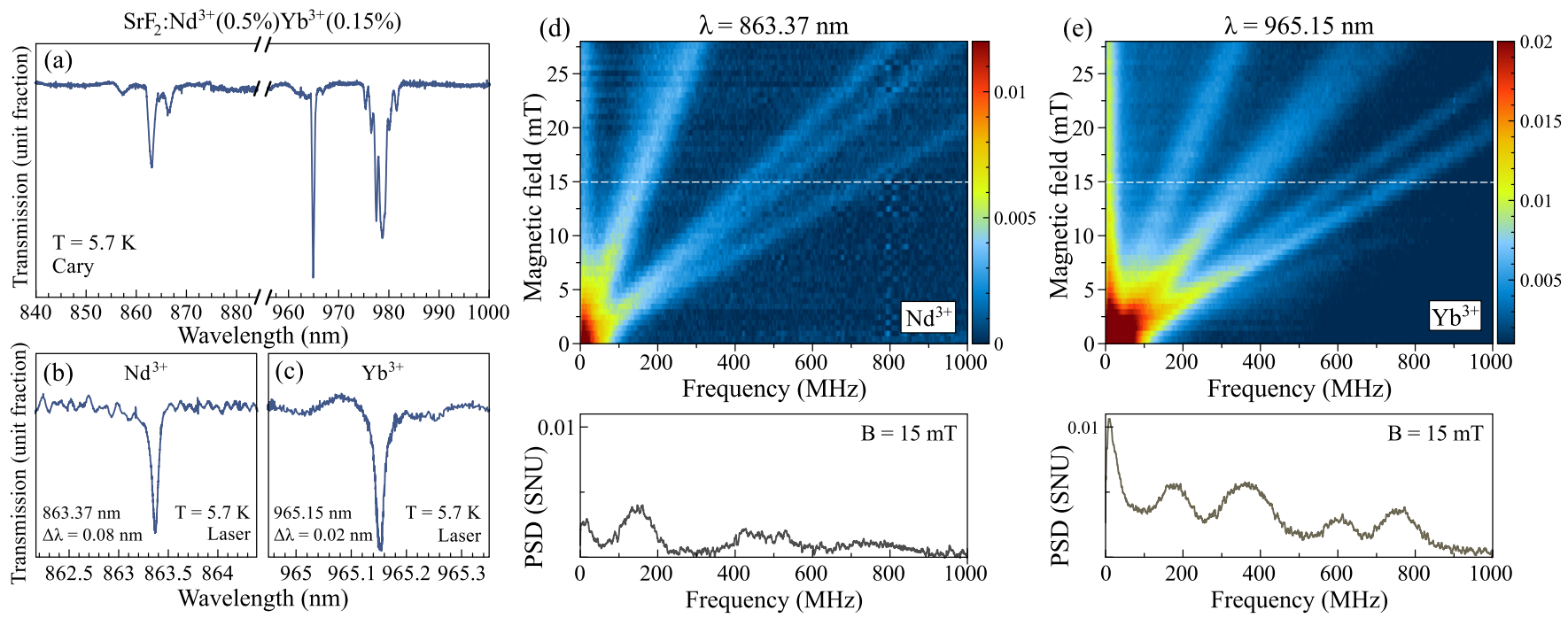

FIG. 3. SN spectra of the studied $\mathrm{SrF}_{2}: \mathrm{Nd}^{3+}(0.5 \%) \mathrm{Yb}^{3+}(0.15 \%)$ crystal. (a) Transmission spectra obtained using a Cary spectrophotometer with a spectral resolution of $0.05 \mathrm{~nm}$. (b) and (c) High-resolution transmission spectra obtained with a tunable Ti:sapphire laser. Laser power is $1 \mathrm{~mW}$. The oscillating background signal is produced by laser etaloning at the sample surfaces. (d) and (e) are color maps demonstrating the magnetic field dependence of the SN power spectrum density (PSD) for the $\mathrm{Nd}^{3+}$ and $\mathrm{Yb}^{3+}$ ions, respectively. The spectra are detected for the same orientation of the crystal with respect to the magnetic field and light propagation directions. The white dashed lines in the color spectra mark the magnetic fields at which the spectra below are taken in shot noise units (SNU). We remind one that the multitude of lines in each figure corresponds to the several groups of magnetically nonequivalent anisotropic centers for each impurity ion.

focused on the sample to a spot of $40 \mu \mathrm{m}$ diameter, using a 200-mm focal-length lens.

Overview measurements of transmission spectra of sample 1 [Fig. 3(a)] were performed with a Cary spectrophotometer providing a spectral resolution of $0.05 \mathrm{~nm}$, in order to identify the spectral features that are suitable for the resonant SN detection. The most convenient $f$ - $f$ transitions were found in the spectral range $860-870 \mathrm{~nm}$ for $\mathrm{Nd}^{3+}$ and $965-980 \mathrm{~nm}$ for $\mathrm{Yb}^{3+}$, in agreement with literature data [28]. For a more detailed characterization, we performed transmission measurements with much higher spectral resolution, using the tunable Ti:sapphire laser [Figs. 3(b) and 3(c)]. Narrow spectral features are observed then in both spectral regions: the $\mathrm{Nd}^{3+}$ ions showed one absorption line at $863.37 \mathrm{~nm}$ with the FWHM $\Delta \lambda=0.08 \mathrm{~nm}$, obtained using a Gaussian fit; the $\mathrm{Yb}^{3+}$ ions demonstrated a strong absorption peak at $965.15 \mathrm{~nm}$ with the width $\Delta \lambda=0.02 \mathrm{~nm}$.

By tuning the laser emission wavelength to the absorption peaks of the corresponding lines we could detect the strongest SN signal [14]. Figures 3(d) and 3(e) show color maps of the magnetic field dependences of the noise power spectra, detected in the Voigt geometry. Examples of these spectra at $B=15 \mathrm{mT}$ are given below these maps for the two used wavelengths and, correspondingly, for the two ion coactivators. The multitude of lines in the SN spectra demonstrates the presence of several groups of magnetically nonequivalent, anisotropic centers, which is typical for the heterovalent substitution of impurity ions. Furthermore, these measurements show the high spectral selectivity of SN spectroscopy, especially in comparison with EPR spectroscopy, where the overall signal from the tested sample volume containing the whole variety of impurity centers is detected.

Detailed experiments were performed using the sample containing a single dopant (sample 2). Figure 4(a) shows the laser transmission spectrum of the sample at $T=5.7 \mathrm{~K}$. We tested the SN signal at all five absorption peaks observed in this spectral region, and managed to detect a strong SN signal only at the peak with $862.69 \mathrm{~nm}$ [Fig. 4(b)]. In the color map, we also observe several lines with different magnetic field dispersions. Figure 4(c) demonstrates an exemplary spectrum of the SN power density in units of the shot-noise power at $B=12 \mathrm{mT}$ for the probe light power of $1 \mathrm{~mW}$. Full information about the symmetry and structure of the impurity centers revealed in the SN spectra can be obtained from their orientational dependence, while the field dependence of the resonance linewidth can provide information about spinrelaxation rates of the impurity ion.

The method used here to demonstrate the SN gain effect implies resonant probing of the system and, in general, cannot be considered as nonperturbative. So, it is interesting to study the probe light power dependence of the magnetic resonance linewidth detected for these conditions. To that end, we measured the light power dependence of the amplitude and width of the peak at $225 \mathrm{MHz}$ [see insets in Fig. 4(c)]. The amplitude power dependence (plotted in log-log scale) demonstrates an initial linear increase followed by saturation, while the linewidth in this range of light power densities remains constant at $13.6 \mathrm{MHz}$. Therefore, we can conclude that the resonant laser light, which probes forbidden electronic transitions of the RE ions, does not noticeably affect their spin dynamics for the used experimental conditions.

\section{CONCLUSIONS}

In summary, we have shown that the effect of spin-noise gain, which should be observable in many paramagnetic impurities with not completely filled electronic shells, could widen the class of objects to which $\mathrm{SN}$ spectroscopy can be 

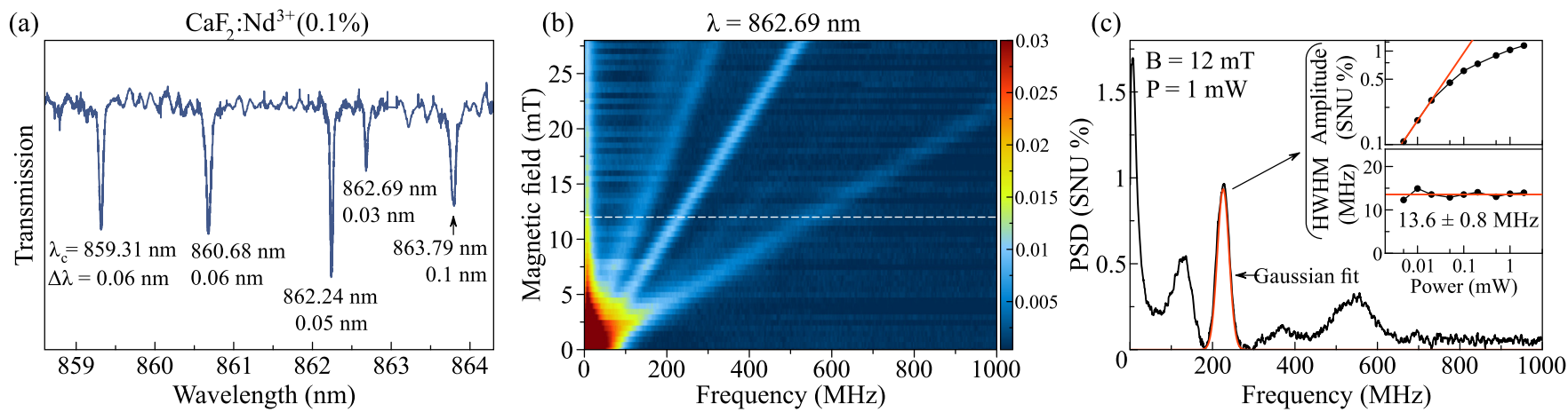

FIG. 4. Spin-noise spectra of the $\mathrm{CaF}_{2}: \mathrm{Nd}^{3+}(0.1 \%)$ crystal. (a) Laser transmission spectrum of the sample at $5.7 \mathrm{~K}$. The oscillations are due to the etaloning effect of the laser by the sample surfaces. (b) Color map of SN spectra detected at the absorption peak $862.69 \mathrm{~nm}$ versus magnetic field. Laser power is $1 \mathrm{~mW}$. (c) Exemplary spectrum at $B=12 \mathrm{mT}$ with the two insets demonstrating the power dependence of the amplitude (log-log scale) and the HWHM (semilogarithmic scale) of the peak marked in the main panel.

applied, and thus enhance the potential of EPR spectroscopy for studying impurity paramagnets. The SN technique provides the EPR spectroscopy with additional degrees of freedom hardly accessible to its classical implementation. The optical excitation and detection provide spectral selectivity of the impurity centers and high spatial resolution both in transverse and in longitudinal directions (three-dimensional tomography). It is important that the $\mathrm{SN}$ measurements can be performed in a wide frequency range which is not restricted by the photodetector bandwidth $[34,35]$. The EPR measurements in SN spectroscopy are not rigidly coupled to any fixed radio frequency, as is usually the case in conventional EPR spectroscopy, and allow one to directly obtain panoramic EPR spectra over a wide spectral range. It is important to emphasize, that the SN signal does not depend explicitly on the spin-system polarization, and the absence (or smallness) of a population difference between spin sublevels does not prevent the SN resonance detection. We believe that these specific features of the SN spectroscopy will essentially advance the present-day magnetic resonance spectroscopy of crystals and glasses containing paramagnetic impurities.

\section{ACKNOWLEDGMENTS}

We acknowledge financial support by the Deutsche Forschungsgemeinschaft through the International Collaborative Research Center TRR 160 (Project A5) and the Russian Foundation for Basic Research (Grant No. 19-52-12054). The theoretical part of the work was supported by the Russian Science Foundation (Project 17-12-01124). I.I.R., G.G.K., and V.S.Z. acknowledge Saint-Petersburg State University for a research grant, Grant No. 51125686.
[1] E. Zavoisky, Spin-magnetic resonance in paramagnetics, J. Phys. E (USSR), 9, 245 (1945).

[2] E. B. Aleksandrov, and V. S. Zapasskii, Magnetic resonance in the Faraday-rotation noise spectrum, Sov. Phys. JETP 54, 64 (1981).

[3] T. Mitsui, Spontaneous Noise Spectroscopy of an Atomic Magnetic Resonance, Phys. Rev. Lett. 84, 5292 (2000).

[4] S. A. Crooker, D. G. Rickel, A. V. Balatsky, and D. Smith, Spectroscopy of spontaneous spin noise as a probe of spin dynamics and magnetic resonance, Nature (London) 431, 49 (2004).

[5] B. Mihaila, S. A. Crooker, D. G. Rickel, K. B. Blagoev, P. B. Littlewood, and D. L. Smith, Quantitative study of spin noise spectroscopy in a classical gas of $41 \mathrm{~K}$, Phys. Rev. A 74, 043819 (2006).

[6] M. Oestreich, M. Römer, R. J. Haug, and D. Hägele, Spin Noise Spectroscopy in GaAs, Phys. Rev. Lett. 95, 216603 (2005).

[7] A. V. Kuhlmann, J. Houel, A. Ludwig, L. Greuter, D. Reuter, A. D. Wieck, M. Poggio, and R. J. Warburton, Charge noise and spin noise in a semiconductor quantum device, Nat. Phys. 9, 570 (2013)

[8] M. M. Glazov and V. S. Zapasskii, Linear optics, Raman scattering, and spin noise spectroscopy, Opt. Express 23, 11713 (2015).
[9] M. Atature, J. Dreiser, A. Badolato, and A. Imamoglu, Observation of Faraday rotation from a single confined spin, Nat. Phys. 3, 101 (2007).

[10] R. Dahbashi, J. Hübner, F. Berski, K. Pierz, and M. Oestreich, Optical Spin Noise of A Single Hole Spin Localized in an (InGa)As Quantum Dot, Phys. Rev. Lett. 112, 156601 (2014).

[11] I. I. Ryzhov, S. V. Poltavtsev, K. V. Kavokin, M. M. Glazov, G. G. Kozlov, M. Vladimirova, D. Scalbert, S. Cronenberger, A. V. Kavokin, A. Lemaître, J. Bloch, and V. S. Zapasskii, Measurements of nuclear spin dynamics by spin-noise spectroscopy, Appl. Phys. Lett. 106, 242405 (2015).

[12] I. I. Ryzhov, G. G. Kozlov, D. S. Smirnov, M. M. Glazov, Y. P. Efimov, S. A. Eliseev, V. A. Lovtcius, V. V. Petrov, K. V. Kavokin, A. V. Kavokin, and V. S. Zapasskii, Spin noise explores local magnetic fields in a semiconductor, Sci. Rep. 6, 21062 (2016).

[13] M. Römer, J. Hubner, and M. Oestreich, Spatially resolved doping concentration measurement in semiconductors via spin noise spectroscopy, Appl. Phys. Lett. 94, 112105 (2009).

[14] V. S. Zapasskii, A. Greilich, S. A. Crooker, Yan Li, G. G. Kozlov, D. R. Yakovlev, D. Reuter, A. D. Wieck, and M. Bayer, Optical Spectroscopy of Spin Noise, Phys. Rev. Lett. 110, 176601 (2013). 
[15] L. Yang, P. Glasenapp, A. Greilich, D. Reuter, A. D. Wieck, D. R. Yakovlev, M. Bayer, and S. A. Crooker, Two-colour spin noise spectroscopy and fluctuation correlations reveal homogeneous linewidths within quantum-dot ensembles, Nat. Commun. 5, 4949 (2014).

[16] G. M. Müller, M. Oestreich, M. Römer, and J. Hübner, Semiconductor spin noise spectroscopy: Fundamentals, accomplishments, and challenges, Physica E 43, 569 (2010).

[17] V. S. Zapasskii, Spin-noise spectroscopy: From proof of principle to applications, Adv. Opt. Photonics 5, 131 (2013).

[18] J. Hübner, F. Berski, R. Dahbashi, and M. Oestreich, The rise of spin noise spectroscopy in semiconductors: From acoustic to GHz frequencies, Phys. Status Solidi B 251, 1824 (2014).

[19] M. M. Glazov, Spin fluctuations of nonequilibrium electrons and excitons in semiconductors, J. Exp. Theor. Phys. 122, 472 (2016).

[20] T. Zhong, J. M. Kindem, E. Miyazono, and A. Faraon, Nanophotonic coherent light-matter interfaces based on rareearth-doped crystals, Nat. Commun. 6, 8206 (2015).

[21] P. Siyushev, K. Xia, R. Reuter, M. Jamali, N. Zhao, N. Yang, C. Duan, N. Kukharchyk, A. D. Wieck, R. Kolesov, and J. Wrachtrup, Coherent properties of single rare-earth spin qubits, Nat. Commun. 5, 3895 (2014).

[22] I. S. Osborne, A rare-earth quantum memory, Science 357, 1366 (2017).

[23] T. Zhong and $\mathrm{Ph}$. Goldner, Emerging rare-earth doped material platforms for quantum nanophotonics, Nanophotonics 8, 2003 (2019).

[24] V. S. Zapasskii, Polarimetry of regular and stochastic signals in magnetooptics, Phys. Solid State 61, 847 (2019).

[25] R. Giri, S. Cronenberger, M. Vladimirova, D. Scalbert, K. V. Kavokin, M. M. Glazov, M. Nawrocki, A. Lemaître, and J.
Bloch, Giant photoinduced Faraday rotation due to the spinpolarized electron gas in an $n$-GaAs microcavity, Phys. Rev. B 85, 195313 (2012).

[26] A. S. Marfunin, Spectroscopy, luminescence and radiation centers in minerals (Springer-Verlag, Berlin/Heidelberg/New York, 1979).

[27] P. Goldner, A. Ferrier, and O. Guillot-Noël, in Handbook on the Physics and Chemistry of Rare Earths (Elsevier B. V., Amsterdam, Oxford, 2015), Vol. 46, pp. 1-78.

[28] R. M. Macfarlane, High-resolution laser spectroscopy of rareearth doped insulators: A personal perspective, J. Lumin. 100, 1 (2002).

[29] A. Abragam and B. Bleaney, Electron Paramagnetic Resonance of Transition Ions (Clarendon, Oxford, 1970).

[30] A. A. Kaplyanskii, Noncubic centers in cubic crystals and their spectra in external fields, J. Phys., Colloq. 28, C4-39 (1967).

[31] S. Cronenberger and D. Scalbert, Quantum limited heterodyne detection of spin noise, Rev. Sci. Instrum. 87, 093111 (2016).

[32] M. Yu. Petrov, A. N. Kamenskii, V. S. Zapasskii, M. Bayer, and A. Greilich, Increased sensitivity of spin noise spectroscopy using homodyne detection in $n$-doped GaAs, Phys. Rev. B 97, 125202 (2018).

[33] S. A. Crooker, J. Brandt, C. Sandfort, A. Greilich, D. R. Yakovlev, D. Reuter, A. D. Wieck, and M. Bayer, Spin Noise of Electrons and Holes in Self-Assembled Quantum Dots, Phys. Rev. Lett. 104, 036601 (2010).

[34] S. Starosielec and D. Hägele, Ultrafast spin noise spectroscopy, Appl. Phys. Lett. 93, 051116 (2008).

[35] G. M. Müller, M. Römer, J. Hübner, and M. Oestreich, Gigahertz spin noise spectroscopy in $n$-doped bulk GaAs, Phys. Rev. B 81, 121202(R) (2010). 